\title{
Características del empleo y bienestar subjetivo en médicos de la Región Metropolitana
}

\author{
JULIA ACUÑA ${ }^{1}$, IRENE SCHIATTINO ${ }^{2, a}$, NINA HORWITZ ${ }^{1, b}$, \\ M. LUZ BASCUÑÁN ${ }^{1, c}$, JUAN P. JIMÉNEZ ${ }^{1}$
}

\section{Characteristics of employment and wellbeing among physicians working in Santiago, Chile}

Background: Profound changes have been observed in medical practice during the last thirty years. This may be understood as a result of the influence of economic variables in health services management, among other probable causes. At the same time, doctors' work has been diversified, and a tendency to work in several paid jobs simultaneously has been observed. Aim: To describe the characteristics of employment in a representative sample of Chilean physicians. Material and Methods: A probabilistic sample of 414 physicians residing in Metropolitan Santiago answered a survey about their number and type of jobs and completed the BISUMED questionnaire that measures the subjective wellbeing of physicians. Results: Forty percent of surveyed physicians had three or more jobs (36.3\% of men and $47.5 \%$ of women). There was a significant inverse association between the number of jobs, general wellbeing and facing medicine from a new perspective. Conclusions: The number of jobs is inversely related to the general wellbeing of physicians.

(Rev Med Chile 2013; 141: 187-193).

Key words: Employment; Physicians; Wellbeing.

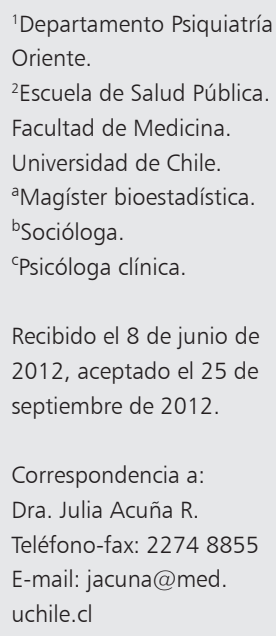

L a profesión médica ha mantenido un sitio reconocido y valorado en la sociedad en diferentes épocas y culturas. Un elemento crucial subyacente a ello, radicaría en la relación de ayuda característica del acto médico. A partir de la segunda mitad del siglo veinte, se produce un cambio sostenido y profundo en la medicina, dado por la triangulación de la relación médico paciente por parte de los prestadores de servicios sanitarios. El giro hacia una visión económica de la salud orienta el foco de interés hacia los costos de la atención, haciendo de ésta un bien transable como cualquier otro en el mercado, produciéndose así un cambio cualitativo en el ejercicio de la profesión. De profesionales que desarrollan su práctica con amplia autonomía, vale decir, con capacidad de decisión acerca de dónde, cuánto y cómo trabajar, el médico se transforma en un empleado de instituciones públicas o privadas donde oferta y demanda son los principales reguladores ${ }^{1-5}$.

Este fenómeno ha sido descrito a partir de la década de 1980-89 en relación a procesos de reformas sanitarias en Europa. Kalble (2005) señala que rentabilidad, eficiencia y ahorro, conceptos rectores del proceso de reforma, afectarían la profesión médica ${ }^{1}$. Un punto crítico señalado por varios autores sería la pérdida de autonomía del médico, como resultado de la creciente necesidad de vigilar y regular los costos de los servicios de salud, lo cual sería entendido como desprofesionalización de la medicina ${ }^{1-7}$. Pero, además significa la trasformación de una relación interpersonal diádica y de carácter privado entre médico y paciente, hacia otra mediada e intervenida por terceros relacionados a la gestión económica del servicio de salud. Por su parte, los pacientes se desenvuelven como clientes que compran servicios, con lo cual se consolida la transformación en la actualmente 
denominada relación clínica, concurriendo en ella otros actores (tanto personas como instituciones), además del cliente y el médico-empleado ${ }^{7-9}$.

Los cambios en la práctica de la medicina han sido ampliamente investigados en diversos sentidos. Entre ellos ha tenido especial interés la relación de diversos aspectos del trabajo con satisfacción/ insatisfacción, con el ejercicio profesional, el bienestar y la salud de los médicos ${ }^{6,10-14}$. Sin embargo, las consecuencias del impacto en la calidad de los servicios, en indicadores económicos y en la satisfacción de los pacientes ha tenido menor atención, a pesar de consistente información que ha señalado que el bienestar, satisfacción, niveles de estrés y salud de los médicos, influyen no sólo a nivel personal, sino también en el desempeño profesional y en las organizaciones sanitarias a través de: errores, mala práctica, satisfacción del paciente, adherencia a tratamiento, productividad, eficiencia, dificultad de reclutamiento, recambio ocupacional, por nombrar algunos. Por lo anterior, el bienestar médico ha sido señalado como indicador de calidad ${ }^{2,15}$.

Chile no ha estado ausente de los cambios descritos en el sector salud ni del impacto de éstos en los médicos. El campo laboral se ha diversificado debido al desarrollo creciente de la salud privada, transitando así de una situación en la cual la principal alternativa laboral estaba en los servicios públicos de salud, a otra, en la cual existen diversos empleadores potenciales tanto públicos como privados quienes ofrecen un mercado laboral múltiple y diverso. Como resultado, se ha advertido una tendencia a ejercer la medicina en varios empleos remunerados simultáneamente ${ }^{16-18}$.

Este estudio es parte de una línea de investigación sobre el bienestar subjetivo de los médicos ante los cambios en la profesión ${ }^{16,17,19-21}$. El objetivo de la presente comunicación, es describir las características del empleo en una muestra representativa de médicos colegiados de la Región Metropolitana de Santiago de Chile. Se evalúa la relación del número de empleos con variables sociodemográficas (edad, género, estado civil), especialidades médicas y dimensiones del cuestionario "Bienestar subjetivo de los médicos BISUMED"20, que se describen en la sección Material y Método.

\section{Material y Método}

Se trata de un estudio transversal descriptivo acerca de las características del empleo de los mé- dicos. La población objetivo estuvo compuesta por los médicos residentes en la Región Metropolitana e inscritos en el registro del Colegio Médico de Chile en el año 2006 (6.707 médicos $)^{20,22}$. Para el presente análisis se utilizó una muestra probabilística estratificada por especialidad, de $414^{\star}$ médicos, considerando un nivel de confianza de $95 \%$ y suponiendo una varianza máxima ${ }^{20,22}$.

La variable "número de empleos durante el último año" define el número de empleos reportado por cada médico encuestado, en un rango de uno a seis empleos. Las especialidades médicas se agrupan en siete grupos: Cirugía, Medicina Interna, Obstetricia y Ginecología, Pediatría, Psiquiatría, Atención Primaria y otras especialidades. El cuestionario BISUMED, que como ya se señaló mide el bienestar subjetivo de los médicos consta de 54 ítems y nueve dimensiones: bienestar general, salud física y emocional, confianza en uno mismo y autonomía, percepción de cambios en el ejercicio de la profesión, espiritualidad y trascendencia, relaciones interpersonales, apoyo y reconocimiento en el trabajo, afrontamiento activo y reflexivo y afrontamiento a través de la revalorización de la medicina y la vocación de servicio.

El análisis estadístico se realizó con el programa STATA 10.0, aplicando pruebas no paramétricas (prueba de Wilcoxon para muestras independientes, análisis de varianza de Kruskal- Wallis, $\chi^{2}$ de asociación y bondad de ajuste). Además, se aplicó el coeficiente de correlación poliserial para describir las correlaciones del número de empleos con las puntuaciones de las dimensiones del cuestionario BISUMED ajustando por género, estado civil, especialidades médicas y tipos de actividad profesional ${ }^{23}$.

\section{Resultados}

\section{Descripción de la muestra}

La Tabla 1 presenta la estructura de la muestra según género y edad. Las mujeres son significativamente más jóvenes que los hombres $(\mathrm{p}=0,00)$. El $85 \%$ de los hombres y $75 \%$ de las mujeres son casados.

La distribución de los profesionales médicos según especialidad se presenta en Tabla 2. La

\footnotetext{
*Submuestra, constituida por encuestas con información completa de variables estudiadas. Diferencias observadas en algunos totales presentados, se debe a datos omitidos.
} 
Empleo y bienestar subjetivo en médicos - J. Acuña et al

especialidad predominante en hombres es cirugía $(25,7 \%)$ y en mujeres, pediatría $(34,6 \%)$.

\section{Variables sociodemográficas}

La distribución de médicos en la muestra total indica que $60 \%$ de ellos tiene uno o dos empleos y que $15,4 \%$ tiene cuatro empleos o más.

Tabla 1. Características de la muestra según edad y género

\begin{tabular}{|lclc|}
\hline Estadísticas & $\begin{array}{c}\text { Hombres } \\
\mathbf{n}=\mathbf{3 0 6}\end{array}$ & $\begin{array}{c}\text { Mujeres } \\
\mathbf{n = 1 0 8}\end{array}$ & $\begin{array}{c}\text { Muestra total } \\
\mathbf{n}=\mathbf{4 1 4}\end{array}$ \\
\hline Mediana & 52 años & 46,5 años & 50 años \\
Rango intercuartílico & 18 años & 18 años & 19 años \\
Mínimo & 25 años & 27 años & 25 años \\
Máximo & 85 años & 78 años & 85 años \\
\hline
\end{tabular}

Tabla 2. Distribución de médicos según especialidades en la muestra total y según género

\begin{tabular}{|c|c|c|c|}
\hline Especialidad & $\begin{array}{l}\text { Hombres } \\
\% \quad \text { (n) }\end{array}$ & $\begin{array}{l}\text { Mujeres } \\
\% \quad \text { (n) }\end{array}$ & \begin{tabular}{l}
\multicolumn{2}{c}{ Total } \\
$\% \quad$ (n)
\end{tabular} \\
\hline Cirugía & $25,7 \quad(78)$ & $9,3 \quad(10)$ & $21,4 \quad(88)$ \\
\hline Medicina interna & $22,0 \quad(67)$ & $18,7 \quad(20)$ & $21,1 \quad(87)$ \\
\hline Pediatría & $18,4 \quad(56)$ & $34,6 \quad(37)$ & $22,6 \quad(93)$ \\
\hline Obstetricia y ginecología & $15,5 \quad(47)$ & $3,7 \quad(4)$ & $12,4 \quad(51)$ \\
\hline Psiquiatría & $3,0 \quad(9)$ & $5,6 \quad(6)$ & $3,7 \quad(15)$ \\
\hline Atención primaria* & $3,6 \quad(11)$ & $7,5 \quad(8)$ & $4,6 \quad(19)$ \\
\hline Otra especialidad & $11,8 \quad(36)$ & $20,6 \quad(22)$ & 14,1 \\
\hline Total & $100,0(304)$ & $100,0(107)$ & $100,0(411)$ \\
\hline
\end{tabular}

*Incluye medicina familiar.
$\mathrm{Al}$ analizar número de empleos en relación con género, se observa una distribución no homogénea tanto al interior del grupo de hombres como de mujeres $(\mathrm{p}=0,00)$. Al comparar por género, sólo hay diferencias significativas en médicos que tienen dos empleos $(\mathrm{p}=0,02)$ (Tabla 3$)$.

Se encontró diferencias significativas en la edad según número de empleos en la muestra total $(\mathrm{p}=0,00)$, situación que se repite sólo en los hombres $(\mathrm{p}=0,01)$. La edad de los médicos varones que tienen un empleo, tiende a ser mayor que la de aquellos que tienen dos o más empleos.

En la Figura 1 se presenta las variaciones de la edad en cada uno de los grupos generados, según número de empleo y género. Al comparar la edad según género, las edades de hombres y mujeres difieren significativamente entre quienes tienen un empleo $(\mathrm{p}=0,01)$, dos empleos, $(\mathrm{p}=0,01)$ y quienes tienen cuatro empleos $(\mathrm{p}=0,00)$.

Finalmente, no se encontraron asociaciones significativas entre el número de empleos y el estado civil, considerando tanto la muestra total como aquella de hombres y de mujeres por separado $(\mathrm{p}>0,41)$.

\section{Especialidades médicas}

No se encontró asociación significativa entre número de empleos y

Tabla 3. Distribución porcentual de médicos según $n^{\circ}$ de empleos y género

\begin{tabular}{|cccccccc|}
\hline No empleos & \multicolumn{2}{c}{ Total } & \multicolumn{2}{c}{ Hombres } & \multicolumn{2}{c|}{ Mujeres } & P \\
& Frecuencia & $\%$ & Frecuencia & $\%$ & Frecuencia & $\%$ & \\
\hline 1 & 108 & 26,47 & 78 & 25,74 & 30 & 28,57 & 0,57 \\
\hline 2 & 142 & 34,80 & 115 & 37,95 & 27 & 25,71 & $0,02^{*}$ \\
\hline 3 & 95 & 23,80 & 70 & 23,10 & 25 & 23,81 & 0,88 \\
\hline 4 & 41 & 10,05 & 27 & 8,91 & 14 & 13,33 & 0,19 \\
\hline 5 & 18 & 4,41 & 11 & 3,63 & 7 & 6,67 & 0,19 \\
\hline 6 & 4 & 0,98 & 2 & 0,66 & 2 & 1,9 & 0,57 \\
\hline Total & 408 & 100 & 303 & 100 & 105 & 100 & \\
\hline
\end{tabular}

*significativo $p \leq 0,05$. 


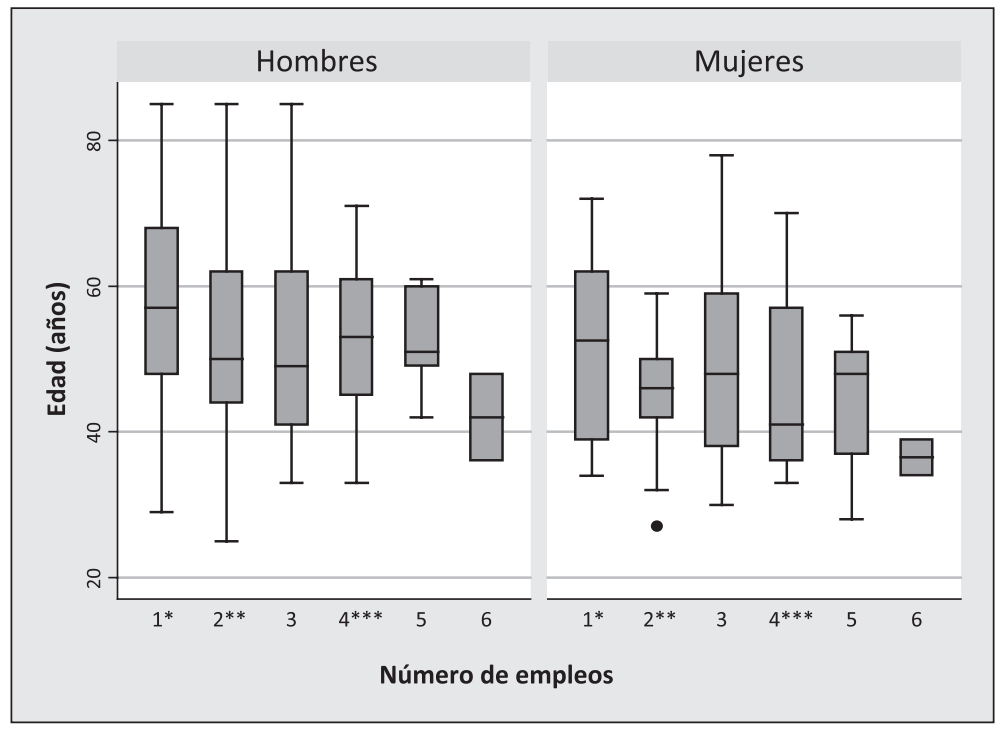

Figura 1. Variación de la edad de los médicos según número de empleos y género. *significativo $p \leq 0,05 .{ }^{* *}$ significativo $p \leq 0,05 . * * *$ significativo $p \leq 0,05$. especialidades en el grupo total $(p=0,46)$, ni en hombres $(\mathrm{p}=0,41)$ y mujeres por separado $(\mathrm{p}=0,50)$ (Tabla 4). Más de $30 \%$ de médicos hombres tienen más de 3 empleos en cada una de las especialidades, excepto en Atención Primaria y Medicina Familiar. Aproximadamente 40\% de las mujeres tiene más de 3 empleos en todas las especialidades.

Al comparar la edad (años) en los grupos generados según número de empleos, en las diferentes especialidades se encontraron diferencias significativas para Cirugía $(\mathrm{p}=0,02)$, Pediatría $(\mathrm{p}=0,01)$, Obstetricia y ginecología $(\mathrm{p}=0,02)$ y Psiquiatría $(\mathrm{p}=0,03)$.

\section{Número de empleos y dimensiones BISUMED}

Como se observa en la Tabla 5, se encontró asociación significativa e inversa entre "Número de empleos" y "Bienestar general" en el grupo total, lo cual se corrobora en mujeres. También se encontró asociación significativa inversa entre "Afrontamiento revalorizando la medicina" en el grupo total, la cual se corrobora en hombres.

El resto de las asociaciones que resultaron significativas, fueron directas.

No se encontraron asociaciones significativas entre estado civil, número de empleos y dimensiones BISUMED.

Algunas especialidades mostraron asociaciones significativas entre número de empleos y las dimensiones BISUMED, las cuales se muestran en
Tabla 4. Distribución de frecuencia de los profesionales médicos según especialidades médicas y número de empleos

\begin{tabular}{|lrrrrrr|}
\hline Especialidad & \multicolumn{5}{c}{$\mathbf{N}^{\circ}$ de empleos * } \\
& $\mathbf{1}$ & $\mathbf{2}$ & $\mathbf{3}$ & $\begin{array}{c}\mathbf{4} \text { o } \\
\text { más* }\end{array}$ & Total \\
Cirugía & 30 & 28 & 16 & 14 & 88 \\
Medicina interna & 23 & 27 & 25 & 12 & 87 \\
Pediatría & 23 & 29 & 21 & 14 & 87 \\
Obstetricia y ginecología & 10 & 22 & 13 & 6 & 51 \\
\hline $\begin{array}{l}\text { Psiquiatría } \\
\text { Atención primaria y }\end{array}$ & 4 & 4 & 4 & 3 & 15 \\
medicina familiar & 3 & 12 & 3 & 1 & 19 \\
Otra especialidad & 15 & 19 & 12 & 12 & 58 \\
Total & 108 & 141 & 94 & 62 & 405 \\
\hline
\end{tabular}

*Se agruparon las clasificaciones 4, 5 o 6 empleos en "4 o más empleos" para aplicar la prueba de asociación $\chi^{2}$.

la Tabla 6. Todas las asociaciones fueron directas a excepción de aquellas observadas entre "Bienestar general" y "Apoyo y reconocimiento en el trabajo" con número de empleos en el grupo de pediatras, las cuales fueron inversas.

\section{Discusión}

Este estudio permitió caracterizar el empleo de los médicos de la Región Metropolitana a través de la variable "número de empleos" y su relación 
Empleo y bienestar subjetivo en médicos - J. Acuña et al

Tabla 5. Asociaciones entre dimensiones del cuestionario BISUMED y $\mathbf{N}^{\circ}$ de empleos en la muestra total y según género

\begin{tabular}{|lccc|}
\hline Dimensión del cuestionario BISUMED & $\begin{array}{c}\text { Hombres }(\mathbf{n}=\mathbf{3 0 3}) \\
\mathbf{R h o *}(\mathbf{p})^{* *}\end{array}$ & $\begin{array}{c}\text { Mujeres }(\mathbf{n}=105) \\
\text { rho }(\mathbf{p})\end{array}$ & $\begin{array}{c}\text { Total }(\mathbf{n}=\mathbf{4 0 8}) \\
\text { rho (p) }\end{array}$ \\
\hline Bienestar general & $-0,06(0,29)$ & $-0,28(0,01)^{* *}$ & $-0,11^{*}(0,04)^{* *}$ \\
\hline Confianza en uno mismo y autonomía & $0,07(0,26)$ & $0,22(0,03)^{* *}$ & $0,11^{*}(0,03)^{* *}$ \\
\hline Espiritualidad y trascendencia & $0,05(0,43)$ & $0,27(0,01)^{* *}$ & $0,11^{*}(0,03)^{* *}$ \\
\hline Afrontamiento revalorizando medicina & $-0,13(0,04)^{* *}$ & $-0,12(0,28)$ & $-0,12^{*}(0,02)^{* *}$ \\
\hline Afrontamiento activo reflexivo & $0,14(0,02)^{* *}$ & $-0,03(0,75)$ & $0,09(0,08)$ \\
\hline
\end{tabular}

${ }^{*}$ rho $=$ coeficiente de correlación poliserial. ${ }^{* *}$ significativo $p \leq 0,05$.

Tabla 6. Asociaciones significativas entre dimensiones del cuestionario BISUMED y $\mathrm{N}^{\circ}$ de empleos en especialidades médicas consideradas en el estudio

\begin{tabular}{|lll|}
\hline Especialidades (n) & Dimensión del cuestionario BISUMED & Rho* $(\mathbf{p})^{* *}$ \\
Pediatría (87) & Bienestar general & $-0,40(0,00)$ \\
& Apoyo y reconocimiento en el trabajo & $-0,23(0,04)$ \\
& Percepción de salud física y emocional & $0,27(0,03)$ \\
& Confianza en uno mismo y autonomía & $0,35(0,00)$ \\
\hline Medicina Interna (87) & Espiritualidad y trascendencia & $0,29(0,01)$ \\
Cirugía (88) & Relaciones interpersonales & $0,33(0,00)$ \\
Psiquiatría (15) & Relaciones interpersonales & $0,68(0,04)$ \\
& Afrontamiento activo reflexivo & $0,51(0,03)$ \\
\hline
\end{tabular}

${ }^{*}$ rho $=$ coeficiente de correlación poliserial; **significativo $p \leq 0,05$.

con variables socio demográficas, especialidades $\mathrm{y}$ dimensiones del instrumento BISUMED acerca del Bienestar subjetivo de los médicos ${ }^{20}$.

La importancia de la variable "número de empleos" es entendida en el presente estudio como una consecuencia de la incorporación de variables económicas en la organización y gestión de servicios sanitarios. Constituye también un indicador de precariedad laboral, característica que afecta crecientemente a la fuerza laboral en general y que tiene efectos sobre la salud. La precariedad laboral ha dado lugar a una definición multidimensional, aunque en términos específicos, se puede constatar que la mayor cantidad de investigaciones recientes sobre este tema se enfocan preferentemente en los de la temporalidad e inestabilidad del empleo, ya que se intensifica la imprevisibilidad y falta de control asociada a la dependencia exclusiva del salario como ingreso ${ }^{24}$.

Los resultados revelan que aproximadamente $40 \%$ de los médicos tiene tres o más empleos, hallazgo algo mayor a lo señalado recientemente por Santander y cols. (2010), quienes informan
$33 \%$ para dicha condición. En nuestro estudio se destaca que $36,3 \%$ de los hombres y $45,7 \%$ de las mujeres declaran tener 3 empleos o más. Esto resulta consistente con lo informado por Santander y cols., para los hombres, quienes encontraron que $37,8 \%$ de ellos reportaba tener tres empleos o más; sólo $17 \%$ de las mujeres en ese estudio reportaron esa condición. Las diferencias encontradas entre las mujeres podrían ser explicadas en parte por la composición de las muestras: la nuestra, con distribución homogénea en los diferentes rangos etáreos, la otra en tanto, con predominio de mujeres jóvenes (48\% de mujeres tenía 34 años o menos), esperándose para estas edades una priorización del rol maternal por sobre el profesional.

En el presente estudio se destaca que el empleo múltiple afecta negativamente el bienestar de las mujeres. Esto podría estar relacionado, entre otros factores, con el hecho que además de los roles profesionales, las mujeres asumen mayoritariamente la responsabilidad de los roles familiares. Santander y cols. (2010), encontraron valoraciones negativas para roles familiares, significativamente 
mayores en mujeres que en hombres; más aún, cerca de $40 \%$ de las mujeres declaró que debería haber elegido otra profesión, diferenciándose en ello significativamente de los hombres ${ }^{18}$. La interferencia de roles como causa de insatisfacción con el ejercicio profesional de médicas ha sido confirmado en diferentes estudios ${ }^{25-28}$. Todo esto se recoge en nuestro estudio en la dimensión Bienestar general de BISUMED, que incluye ítems de bienestar subjetivo vinculados a la vida en general y a la vida laboral, como por ejemplo, el nivel de satisfacción con lo realizado en los últimos años, satisfacción con el trabajo, percepción de progreso en la vida y logro de metas ${ }^{20}$.

Es destacable mencionar que las mujeres con mayor número de empleos mostraron también mayor "Espiritualidad y trascendencia". Asimismo, ellas mostraron valores significativamente más altos que los hombres en "Confianza en uno mismo y autonomía”. A este respecto, es necesario aclarar que el sentido de los ítems de esta dimensión en el Cuestionario BISUMED se refiere a atributos personales, estrategias de afrontamiento y autonomía en general y no a autonomía específica en el contexto laboral ${ }^{20}$. Se podría postular que las mujeres desplegarían estrategias de afrontamiento de tipo espiritual ante una situación laboral estresante $^{29}$, o bien, que a partir de atributos personales como confianza en uno mismo y autonomía, las mujeres médicas podrían aceptar trabajos múltiples, pero sin embargo, no sería suficiente para alcanzar un sentido de bienestar.

$\mathrm{Al}$ examinar las especialidades médicas se observaron asociaciones significativas importantes entre número de empleos y dimensiones BISUMED. Los pediatras muestran un perfil particular a través de cuatro de las nueve dimensiones BISUMED. Contrario a lo esperado y a lo observado en el grupo de mujeres, a mayor número de empleos ellos revelan mayor bienestar. Se podría plantear que siendo en nuestra muestra los pediatras mayoritariamente hombres, podrían estar menos afectados por los conflictos de rol. Otra explicación posible sería que "Confianza en sí mismos y autonomía” y "Apoyo y reconocimiento en el trabajo" se potenciarían como factores protectores como se ha señalado en diversos trabajos ${ }^{11-14}$. Pero además perciben que su "Salud física y emocional" sería mejor. Esto resulta un hallazgo inusual entre los médicos, quienes tienden a preocuparse poco y tarde de su propia salud ${ }^{15}$.
En general, las asociaciones entre especialidades, empleo y bienestar abren la posibilidad de plantear dos hipótesis. En una se postularía que el especialista usaría como estrategias de afrontamiento habilidades desarrolladas naturalmente en su práctica profesional; en la otra, que serían características personales, incluyendo estrategias de afrontamiento intrínsecas las que influirían en la elección de la especialidad. Esta interrogante escapa a las posibilidades metodológicas de respuesta del presente estudio lo cual podría ser abordado en el futuro.

Este trabajo ha permitido una interesante y necesaria descripción exploratoria de las características del empleo médico en la Región Metropolitana y de su relación con el bienestar subjetivo y otras percepciones de estos profesionales. Se constata altas tasas de empleo múltiple, pero también se aprecia que las especialidades médicas y el género revelan a través de la subjetividad de los médicos, que estilos personales o habilidades propias de la especialidad podrían llegar a constituirse en factores protectores o de riesgo frente condiciones adversas como el empleo múltiple.

Una limitación del estudio lo remite a la constitución de la muestra. A pesar de su representatividad derivada de la rigurosidad metodológica empleada, mantiene las restricciones propias del universo que representa, único conocido y disponible en su momento (registros del Colegio Médico, año 2006). Quizás pudiera considerarse también una limitación, la focalización del estudio en los médicos, explicado esto por la línea de investigación de la cual forma parte, centrada en la percepción de estos profesionales ante cambios observados en la práctica profesional durante las últimas décadas. Sin embargo, el presente estudio puede servir de referencia para otros en los cuales se amplíe el foco a otros profesionales de la salud e incluso, a otras profesiones del área de servicios.

Finalmente, cabe considerar la pertinencia de una actualización del estudio, dado que los cambios señalados se han ido consolidando y el universo estudiado se ha ampliado por el número creciente de médicos inscritos en el Colegio Médico de Chile.

\section{Referencias}

1. Kalble K. Between professional autonomy and economic orientation-The medical profession in a changing health care system. GMS Psychosoc Med. 2005: Doc01. Dispo- 
nible en http://www.egms.de/en/journals/psm/2005-2 psm00010.shtml [consultado el 15 de julio de 2011].

2. Arnetz B. Psychosocial challanges facing the physicians of today. Soc Sci Med 2001; 52: 203-13.

3. Edwards N, Kornacki NJ, Silversin J. Unhappy doctors: what are the causes and what can be done? BMJ 2002; 324: 835-8.

4. Ham Ch, Alberti K. The medical, the public, and the government. BMJ 2002; 324: 838-42.

5. Magee M, Hojat M. Impact of health care system on physicians'discontent. Journal of Community Health 200; 26: 357-65.

6. Landon B, Reschovsky J, Blumenthal D. Changes in career satisfaction among Primary Care and Specialist Physicians 1997-2001. JAMA 2003; 289: 442-9.

7. Bascuñán ML. Cambios en la relación médico-paciente y nivel de satisfacción de los médicos. Rev Med Chile 2005; 133 (1): 11-6.

8. Lázaro J, Gracia D. La relación médico-enfermo a través de la historia. An Sist Sanit Navar 2006; 29 (3): 7-17.

9. Forsberg E, Axelsson R, Arnetz B. Performance-based reimbursement in health care. Euro J Public Health. 2002; 12 (1): 44-50. Disponible en http://www.ncbi.nlm. nih.gov/pubmed/ [consultado el 30 de agosto de 2011]

10. Lee H-Y, Park SE, Park E-C, Hahm M-I, Cho WH. Job satisfaction and trust in Health Insurance Review Agency among Korean physicians. Health Policy. 2008; 87: 249-257. Disponible en http://www.sciencedirect. com/science/article/pii/ [consultado el 15 de julio de 2011].

11. Wada K, Arimatsu M, Higashi T, Yoshikawa T, Oda S, Taniguchi H, et al. Physician job satisfaction and working conditions on Japan. J Occup Health. 2009; 51: 261266. Disponible en http://www.jstage.jst.go.jp/article/ joh/51/3/51 [consultado el 15 de julio de 2011].

12. Levine R, Harrison R, Mechaber H, Phillips Ch, Gallagher T. Professional characteristics on job satisfaction among SGIM members: a comparison of part-time and full-time physician members. J Gen Inter Med 2008; 23 (8): 1218-21.

13. Stoddard J, Hargraves J, Reed M, Vratil A. Managed care, professional autonomy and income. Effects on physician career satisfaction. J Gen Inter Med 2001; 16: 675-84.

14. Scheurer D, McKean S, Miller J, Wetterneck T. U.S. Physician satisfaction: a systematic review. J Hosp Med 2009; 4 (9): 560-70. Disponible en: http://onlinelibrary. wiley.com/ [consultado el 30 de agosto de 2011].

15. Wallance J, Lemaire J, Ghali W. Physician wellness: a missing quality indicator. The Lancet 2009; 374: 1715-20.
16. Jiménez J. Cambios en el entorno del trabajo médico. Rev Med Chile 2004; 132: 637-42.

17. Horwitz N. El cambio de la práctica médica: Desafíos psicosociales para la profesión. Rev Med Chile 2004; 132: 768-72.

18. Santander S, Sánchez J, Zapata L, Sánchez S. Situación laboral, económica y académica de los médicos de la Región Metropolitana. Cuad Méd Soc 2010; 50 (1): 24 38.

19. Jiménez JP. Bienestar y satisfacción de los médicos con el ejercicio de su profesión en un sistema de salud en proceso de reforma. Rev Med Chile 2004; 132: 635-6.

20. Horwitz N, Schiattino I, Bascuñán ML, Acuña J, Jiménez JP. Validación de una escala para medir el bienestar subjetivo de los médicos (BISUMED). Rev Med Chile (2010); 138: 1084-90.

21. Bascuñan ML, Horwitz N, Schiattino I, Acuña J, Jiménez JP. Percepción de cambios en la práctica médica y estrategias de afrontamiento. Rev Med Chile 2011; 139 (10): 1305-12.

22. Departamento de Economía, Universidad de Chile. Centro MICRO DATOS. Informe final Encuesta Bienestar Subjetivo de los Médicos, Santiago, Chile. Septiembre 2007.

23. Olsson U, Drasgow F, Dorans N. The polyserial correlation coefficient. Psychometrika 1982; 47 (3): 337-51.

24. Encuesta Nacional de Empleo, Trabajo, Salud y Calidad de Vida de los Trabajadores y Trabajadoras de Chile (ENETS 2009-2010). Departamento de Epidemiología, Ministerio de Salud http://www.saludytrabajo.cl/wpcontent/uploads/2011/09/PRECARIEDAD_BAJA.pdf [consultado 2/05/2012].

25. Changing society: changing role of doctors. (Editorial). BMJ 2000; 320: 1417-8.

26. Caniano D, Sonnino R, Paolo A. Keys to career satisfaction: insights from a survey of women pediatric surgeons. J Pediatr Surg 2004; 39 (6): 984-90.

27. Fuss I, Nübling M, Hasselhorn H-M, Schappach D, Rieger M. Working conditions and work- family conflict in German hospital physicians: psychosocial and organisational predictors and consequences. BMC Public Health 2008; 8: 353. doi:10.1186/147-2458/353. Disponible en http://www.biomedcentral.com/14712458/8/353 [consultado el 30 de agosto de 2011].

28. Santander S, Sánchez J, Zapata L, Sánchez S. Aspectos de la vida familiar de los médicos de Santiago de Chile. Cuad Méd Soc (Chile) 2010; 50 (4): 296-306.

29. Kim Y, Seidlitz L. Spirituality moderates the effect of stress on emotional and physical adjustment. Personality and individuals differences 2002; 32: 1377-90. 